\title{
VALUE OF MATRIX METALLOPROTEINASE AS A PREDICTOR OF OUTCOME OF ACUTE MYOCARDIAL INFARCTION
}

\author{
El Shaeer M. H., Abd El Aziz T. A., Hussein Y. M.*, \\ and Shimaa W. M. * \\ cardiology \& Biochemistry* Departments Faculty of Medicine Zagazig University
}

\begin{abstract}
Background: Following acute myocardial infarction (AMI) many cytokines and proteolytic enzymes are released. Among these, matrix metalloproteinases (MMPs) are important proteolytic enzymes that lead to degradation of the extracellular matrix and to change in cardiomyocytes in both infracted and noninfarcted myocardium. This process is known as cardiac remodelling. It has been demonstrated that more than one type of MMP is present in the circulation after cardiomyocye injury. A number of studies have demonstrated the correlation between these MMP levels and the severity of coronary lesion, the prognosis of left ventricular dimension and the survival rate following AMI.

Aim of the work To assess the usefulness of matrix metalloproteinase and other factors as predictors of outcome of patients with acute myocardial infarction.

Patients and methods: This study was done in Cardiology department and biochemistry department, Zagazig University on 92 patients; 75 males (81.5\%)) 17 females (18.5\%) with acute myocardial infarction. Patients were divided into 4 groups ; group I(Revascularization),group II (Morbidity),group III(Mortality),group IV(Control) All patients were subjected to full laboratory examination and Plasma MMP-3 was measured, wall motion score index(WMSI), systolic and diastolic function were assessed by echocardiography pre-discharge and after 6 months .

Results: MMP-3 peaked prior to discharge. MMP-3 was associated with patient cholesterol $(\mathrm{p}<0.001)$, triglyceride $(\mathrm{p}<0.05)$,WMSI $(\mathrm{p}<0.05)$ and Gensini score $(\mathrm{p}<0.001)$. MMP-3 inversely correlated with fractional shortening at follow-up ( $\mathrm{p}=0.019)$, Ejection fraction and Isovolumic relaxation time In the 37 patients who had complications or died, MMP-3 was higher $(\mathrm{p}<0.001)$. And subjects with levels above optimum cut off identified via ROC curve were more likely to suffer a clinical event $(\mathrm{p}=0.000)$.

Conclusion: MMP-3 is associated with left ventricular dysfunction, adverse left ventricular remodelling and prognosis after AMI.

Key words: matrix metalloproteinase , acute myocardial infarction.
\end{abstract}

\section{INTRODUCTION}

A cute myocardial infarction is currently one of the most important health problems in many countries around the world.

Risk assessment and evaluation of predictors of acute myocardial infarction is an important part of the initial and ongoing evaluation of the patient. It can determine the patient's prognosis and likely response to various treatments, guide decisions as to which therapeutic approach is most appropriate ${ }^{(\mathbf{1})}$.

Following acute myocardial infarction (AMI) many cytokines and proteolytic enzymes are released. Among these, matrix metalloproteinases (MMPs) are important proteolytic enzymes that lead to degradation of the extracellular matrix and to change in cardiomyocytes in both infracted and noninfarcted myocardium. This process is known as cardiac remodelling. It has been demonstrated that more than one type of MMP is present in the circulation after cardiomyocye injury. A number of studies have demonstrated the correlation between these MMP levels and the severity of coronary lesion, the prognosis of left ventricular dimension and the survival rate following $\mathrm{AMI}^{(3)}$.

The matrix metalloproteinases are zink dependent endopptidases with varying substrate specificity and the capacity to degrade many components of the cardiac extracellular matrix ${ }^{(2)}$.
MMPs have also been proposed as a possible novel prognostic indicator for myocardial infarction patients ${ }^{(3)}$.

\section{AIM OF THE WORK}

To assess the usefulness of matrix metalloproteinase as a predictor of outcome of patients with acute myocardial infarction.

\section{PATIENTS AND METHODS}

We conducted a prospective study. We enrolled 92 patients with AMI admitted to the Coronary Care unit (CCU) between December 2011 to April 2013. The diagnosis was based on symptoms consistent with AMI in conjunction with appropriate, dynamic ECG changes (ST segment elevation, STEMI, $89.1 \%$ ) or ST segment/T wave changes (NSTEMI, 10.9\%) and elevation in plasma markers of myocardial necrosis (creatine kinase or troponin I). They were devided into 4 groups:

- Group I: (Revasvularization group): includes 15 patients who had undergone revascularization (percutanous coronary intervension PCI or coronary artery bypass graft CABG), who didn't have any complications.

- Group II(morbidity group): includes 23 patients who had complications in the form of (heart failure, arrhythmia <AF,VT and heart block $>\mathrm{LV}$ thrombus, myocardial aneurysm, cardiogenic shock, cardiac arrest, reinfarction). 
- $\quad$ Group III(mortality group): includes 14 patients who are died.

- Group IV(control group): includes 40 patients who were not revascularized, didn't have complications or died.

\section{Exclusion criteria:}

1. Patients with unstable angina.

2. Patients with congenital, rheumatic heart disease or cardiomyopatyh .

3. Patients with malignant tumor, inflammatory or connective Tissue disease.

Our patients were subjected to the following; complete history taking, Thorough history taking and physical examination and assessed as regard TIMI risk score, Killip class, GRACE score. With special emphasis on age, sex, risk factors including (hypertension, diabetes mellitus, smoking).

12 lead surface electrocardiogram (ECG), full laboratory analysis, including (Plasma matrix metalloproteinase(MMP) concentration by ELISA, Plasma MMP-3 was measured using a commercially available kit (RayBio® Human MMP-3 ELISA Kit ). The lower limit of detection was $14.7 \mathrm{ng} / \mathrm{ml}$. CPk, CK-MB, Tnt, lipid profile, RBs, liver \& Kidney functions and complete blood picture).

Resting complete transthoracic

Echocardiographic evaluation was carried out immediately prior to discharge and at follow-up. Using the commercially available equipment, the digital ultrasound system (HP5500 , Sonos Ultrasound) with a $2-$ to $3-\mathrm{MHz}$ transducer.

M-mode, 2-dimensional and Doppler echocardiographic assessment was performed for all patients.

Examinations were done with the patient in left semi- lateral position; utilizing left parasternal long axis \& short axis views, apical 4, apical 5 and apical 2 chamber views.

A) Two-Dimensional echocardiography to evaluate:

Left ventricle as regard global systolic performance, Segmental wall motion analysis, systolic wall thickening and ejection fraction.

B) M-mode echocardiography to measure:

Left atrium dimension.

Left ventricular end systolic and end diastolic dimensions, wall thickness and fractionl shortening.

C) Doppler echocardiography to measure the early peak filling velocity $\mathrm{E}$ wave and the late peak filling velocity A wave, E/A ratio and deceleration time and isovolumic relaxation time (IVRT).

The following measures were stressed upon and selected for analysis:
1- Left ventricular systolic function:

Ejection fraction (EF\%):

a. It was calculated from the formula:

$$
\mathrm{EF}=\frac{\mathrm{EDV}-\mathrm{ESV} \times 100}{\mathrm{EDV}}
$$

Normally it is $50-70 \%{ }^{(4)}$.

b. Ejection fraction is also measured by area length method (modified Simpson's method (method of diks)

Left ventricular systolic dysfunction is defined as EF less than $50 \%$.

Regional wall motion analysis: Left ventricular wall motion was analyzed according to the 16 segment model of the American society of echocardiography .

A segmental wall motion was graded as follow: Normal $=1$, Hypokinetic $=2$, Akinetic $=3$, Dyskinetic $=4$, Aneurysmal $=5$

Wall motion score index was obtained by dividing the sum of individual visualized segments score by the number of visualized segments ${ }^{(5)}$.

-Left ventricular diastolic function:

It was obtained from Doppler examination of mitral valve flow pattern. The transducer was positioned in the apical 4- chamber view. The sample volume marker was positioned at the level of the tips of the mitral valve leaflets. All the patients were in sinus rhythm.

Transmitral flow pattern had shown two distinct peaks, representing an initial rapid early inflow velocity wave (E-wave) and late diastolic or atrial velocity wave (A-wave).

The following parameters were measured.

E-wave maximum velocity. Normally $=70 \pm 30$ $\mathrm{cm} / \mathrm{sec}$. A-wave maximum velocity. Normally $=$ $50 \pm 20 \mathrm{~cm} / \mathrm{sec}$. E/A ratio obtained by dividing the maximum velocity of E-wave by maximum velocity of A-wave. Normally $=1.461 \pm 0.461{ }^{(4)}$.

Iso-volumic Relaxation Time (IVRT), was measured as the interval from the end of the aortic flow to the onset of mitral inflow with the transducer in apical 5- chamber view with the sample volume marker midway between mitral valve annulus and left ventricular outflow tract. Normally $=100 \pm 20 \mathrm{msec}^{(\boldsymbol{(})}$.

- Follow up of all patients after 6 months for complications (acute heart failure, arrhythmia (AF,VT and heart block), LV thrombus,myocardial aneurysm, cardiogenic shock, cardiac arrest, cardiac events (reinfarction, revascularization or death). And follow up echocardiography.

And for the patients who had done coronary angiography were assessed by Gensini score.

Statistical Analysis: 
Continuous variables are summarized as mean \pm SD. Categorical variables were compared by Chi-squre.

The 1-way analysis of variance followed by Scheffé $\mathrm{F}$ tests were used for numeric variables where appropriate, while paired-t test was used to compare the means of two variables for a single group, it computes the differences between values of the two variables for each case. Correlation between quantitative data calculated by pearsons' correlation. We considered results significant when $P$ value was less than 0.05 . Optimal cut-off values of MMP-3 was obtained from receiver operating characteristic curves. All was calculated SPSS version 12 software program

\section{RESULTS}

Our prospective study had been carried out in cardiology department and biochemistry department, faculty of Medicine, Zagazig university during the period from December 2011 to April 2013.The study group consists of 92 patients presented with acute myocardial infarction, subdivided into 4 groups: Table (1) showed the descriptive analysis of the studied patients. The mean age of the patients was $(57.14 \pm$ $10.85), 81.5 \%$ of the patients were males and $18.5 \%$ of the patients were females. Smoking was the most frequent risk factor in the studied patients $(48.9 \%)$ followed by hypertension $(42.4 \%)$ and diabetes mellitus $(34.8 \%)$.

\section{Table (1): Demographic data \&risk factors of the studied groups}

The statistical analysis shows no significant difference in the age between the 4 groups $(\mathrm{P}=>0.05)$.

Table (1): Demographic data \&risk factors of the studied groups

\begin{tabular}{|c|c|c|c|c|c|c|c|c|c|c|c|}
\hline \multirow[t]{2}{*}{ Variable } & \multirow{2}{*}{\multicolumn{2}{|c|}{$\begin{array}{c}\begin{array}{c}\text { Group } \\
\text { I } \\
(\mathbf{n}=\mathbf{1 5})\end{array} \\
\mathbf{X} \pm \mathbf{S D}\end{array}$}} & \multirow{2}{*}{\multicolumn{2}{|c|}{$\begin{array}{c}\begin{array}{c}\text { Group } \\
\text { II } \\
(\mathrm{n}=23)\end{array} \\
\mathbf{X} \pm \text { SD }\end{array}$}} & \multirow{2}{*}{\multicolumn{2}{|c|}{$\begin{array}{c}\begin{array}{c}\text { Group } \\
\text { III } \\
(\mathrm{n}=14)\end{array} \\
\mathbf{X} \pm \text { SD }\end{array}$}} & \multirow{2}{*}{\multicolumn{2}{|c|}{$\begin{array}{r}\begin{array}{r}\text { Group } \\
\text { IV } \\
(\mathbf{n}=\mathbf{4 0})\end{array} \\
\mathbf{X} \pm \mathrm{SD}\end{array}$}} & \multirow{2}{*}{\multicolumn{2}{|c|}{ Total }} & \multirow[t]{2}{*}{ P-Value } \\
\hline & & & & & & & & & & & \\
\hline \multirow[t]{2}{*}{ Age } & \multicolumn{2}{|c|}{$55.2 \pm 11.6$} & \multicolumn{2}{|c|}{$55.5 \pm 10.6$} & \multicolumn{2}{|c|}{$61.7 \pm 11.5$} & \multicolumn{2}{|c|}{$57.1 \pm 10.3$} & \multicolumn{2}{|c|}{$57.1 \pm 10.8$} & $>0.05$ \\
\hline & $\mathbf{N}$ & $\%$ & $\mathbf{N}$ & $\%$ & $\mathbf{N}$ & $\%$ & $\mathbf{N}$ & $\%$ & $\mathbf{N}$ & $\%$ & \\
\hline \multirow{2}{*}{ Gender } & 14 & 93.4 & 21 & 91.3 & 6 & 42.8 & 33 & 82.5 & 74 & 80.5 & \multirow{2}{*}{$<0.05$} \\
\hline & 1 & 6.6 & 2 & 8.7 & 8 & 57.1 & 7 & 17.5 & 18 & 19.5 & \\
\hline HTN & 4 & 26.7 & 11 & 47.8 & 8 & 57.1 & 16 & 40.0 & 39 & 42.4 & $>0.05$ \\
\hline$\overline{D M}$ & 4 & 26.7 & 10 & 43.5 & 8 & 57.1 & 10 & 25.0 & 32 & 34.8 & $>0.05$ \\
\hline Smokers & 10 & 66.7 & 12 & 52.2 & 1 & 7.1 & 22 & 55.0 & 45 & 48.9 & $<0.05$ \\
\hline
\end{tabular}

Gender: The statistical analysis shows significant difference in the gender between 4groups $(\mathrm{p}<$ $0.05)$.

Hypertension: The statistical analysis shows no significant difference in the presence of hypertension between the 4 groups $(\mathrm{P}=>0.05)$.
Diabetes Mellitus The statistical analysis shows no significant difference in the presence of diabetes mellitus between the 4 groups $(\mathrm{P}=>0.05)$.

Smoking: The statistical analysis showed that there were significant differences in the presence of smoking between the 4 groups $(\mathrm{p}<0.05)$.

Table (2) showed comparison between the studied groups as regard MMP-3

Table ( 2 ): comparison between the four groups as regard MMP-3 level.

\begin{tabular}{|c|c|c|c|c|c|c|}
\hline \begin{tabular}{c}
\multicolumn{1}{c}{ Groups } \\
MMP-3
\end{tabular} & $\begin{array}{c}I \\
N=15\end{array}$ & $\begin{array}{c}\text { II } \\
N=23\end{array}$ & $\begin{array}{c}\text { III } \\
N=14\end{array}$ & $\begin{array}{c}\text { IV } \\
\mathrm{N}=40\end{array}$ & $\mathbf{F}$ & $\mathbf{P}$ \\
\hline \multicolumn{7}{|l|}{ MMP (ng/ml) } \\
\hline Mean \pm SD & $\begin{array}{c}25.25 \\
\pm 17.68 \\
\end{array}$ & $\begin{array}{c}85.68 \\
\pm 29.60 \\
\end{array}$ & $\begin{array}{c}69.11 \\
\pm 31.23 \\
\end{array}$ & $\begin{array}{c}65.80 \\
\pm 21.79 \\
\end{array}$ & 4.519 & $<0.05$ \\
\hline$\overline{\text { Range }}$ & \multicolumn{3}{|c|}{$34.30-93.8026 .30-150.026 .6-150.0$} & $30.60-150.0$ & & \\
\hline
\end{tabular}

The statistical analysis showed that there were significant difference between groups ( $\mathrm{p}$ value $<0.05$ ), which was higher in group II followed by group III then IV and I. 


\section{Hospitalization echocardiographic data in the 4} groups: table(3)

WMSI (1): The statistical analysis shows highly significant difference between groups $(\mathrm{p}<0.001)$, which was higher in group III followed by group II then I and IV.

FS (1): The statistical analysis shows significant difference between groups $(\mathrm{p}<0.001)$, which was lower in group III followed by group II then I and IV.

EF (1): The statistical analysis shows significant difference between groups $(p<0.05)$, which was lower in group III followed by group II then I and IV.

LAD (1): The statistical analysis shows no significant difference between groups ( $p>0.05$ ).

E/A (1): The statistical analysis shows significant difference between groups $(\mathrm{p}<0.05)$.

Deceleration time (1): The statistical analysis shows no significant difference between groups $(\mathrm{p}>0.05)$.

IVRT (1): The statistical analysis shows significant difference between groups $(\mathrm{p}<0.05)$.

Table (3): $\quad$ Showed echocardiographic data in the 4 studied groups during hospitalization.

\begin{tabular}{|c|c|c|c|c|c|c|}
\hline Variable & $\begin{array}{c}\begin{array}{c}\text { Group } \\
\text { I } \\
(n=15)\end{array} \\
X \pm \text { SD }\end{array}$ & $\begin{array}{c}\begin{array}{c}\text { Group } \\
\text { II } \\
(\mathrm{n}=23)\end{array} \\
\mathbf{X} \pm \text { SD }\end{array}$ & $\begin{array}{c}\begin{array}{c}\text { Group } \\
\text { III } \\
(n=14)\end{array} \\
\mathbf{X} \pm \text { SD }\end{array}$ & $\begin{array}{c}\text { Group } \\
\text { IV } \\
(\mathbf{n}=\mathbf{4 0}) \\
\mathrm{X} \pm \mathrm{SD}\end{array}$ & $\begin{array}{r}\text { Total } \\
\mathrm{X} \pm \text { SD }\end{array}$ & P-Value \\
\hline WMSI (1) & $1.35 \pm 0.12$ & $1.46 \pm 0.14$ & $1.54 \pm 0.23$ & $1.33 \pm 0.15$ & $1.39 \pm 0.17$ & $<0.001$ \\
\hline$\overline{\text { FS (\%) (1) }}$ & $31.2 \pm 4.6$ & $29.0 \pm 5.6$ & $26.4 \pm 7.4$ & $33.0 \pm 5.7$ & $30.7 \pm 6.2$ & $<0.05$ \\
\hline $\mathbf{E F}(\%)(1)$ & $57.8 \pm 7.2$ & $55.4 \pm 9.7$ & $51.2 \pm 13.2$ & $60.9 \pm 8.6$ & $57.5 \pm 10.0$ & $<0.05$ \\
\hline $\mathbf{L A D}(\mathrm{mm})(\mathbf{1})$ & $36.2 \pm 4.2$ & $38.3 \pm 4.0$ & $37.8 \pm 4.2$ & $36.0 \pm 4.5$ & $36.9 \pm 4.3$ & NS \\
\hline E/A ratio (1) & $0.95 \pm 0.52$ & $0.94 \pm 0.5$ & $1.41 \pm 0.84$ & $0.93 \pm 0.41$ & $1.01 \pm 0.56$ & $<0.05$ \\
\hline Dec. time $(\mathrm{msec})(1)$ & $198 \pm 106$ & $200 \pm 49$ & $186 \pm 59$ & $189 \pm 44$ & $193 \pm 61$ & NS \\
\hline $\begin{array}{l}\text { IVRT(msec) (1) } \\
\text { (1) }\end{array}$ & $116 \pm 24.4$ & $101 \pm 13.4$ & $95.4 \pm 18.4$ & $108 \pm 16.2$ & $106 \pm 18.4$ & $<0.05$ \\
\hline
\end{tabular}

$\mathrm{X} \pm$ SD: mean and standard deviation, WMSI: wall motion score index, FS: fractional shortening, EF: left ventricular ejection fraction, LAD: left atrial dimension, Dec. time: deceleration time , IVRT: isovolumic relaxation time.

Follow up (after 6 months) echocardio-graphic data in the 4 groups: table (4)

WMSI (2): The statistical analysis shows highly significant difference between groups ( $<<0.001$ ), which was higher in group II followed by group I then IV.

FS (2): The statistical analysis shows highly significant difference between groups $(\mathrm{p}<0.001)$, which was lower in group III followed by group II then I and IV.

EF (2): The statistical analysis shows highly significant difference between groups $(\mathrm{p}<0.001)$, which was lower in group III followed by group II then I and IV.

LAD (2): The statistical analysis shows highly significant difference between groups $(\mathrm{p}<0.001)$, which was increased in group III followed by group II then I and IV.

E/A (2): The statistical analysis shows significant difference between groups $(\mathrm{p}<0.05)$.

Deceleration time (2): The statistical analysis shows no significant difference between groups $(\mathrm{p}>0.05)$.

IVRT (2): The statistical analysis shows significant difference between groups $(\mathrm{p}<0.05)$. 
Table (4): $\quad$ Showed echocardiographic data after 6 months (follow up) in the 4 studied groups

\begin{tabular}{|c|c|c|c|c|c|c|}
\hline Variable & $\begin{array}{c}\begin{array}{c}\text { Group } \\
\text { I } \\
(\mathbf{n}=\mathbf{1 5})\end{array} \\
\mathbf{X} \pm \text { SD }\end{array}$ & $\begin{array}{c}\begin{array}{c}\text { Group } \\
\text { II } \\
(\mathrm{n}=23)\end{array} \\
\mathbf{X} \pm \mathbf{S D}\end{array}$ & $\begin{array}{c}\begin{array}{c}\text { Group } \\
\text { III } \\
(\mathrm{n}=14)\end{array} \\
\mathbf{X} \pm \text { SD }\end{array}$ & $\begin{array}{r}\begin{array}{r}\text { Group } \\
\text { IV } \\
(\mathbf{n}=\mathbf{4 0})\end{array} \\
\mathbf{X} \pm \text { SD }\end{array}$ & Total & P-Value \\
\hline WMSI (2) & $1.34 \pm 0.14$ & $1.53 \pm 0.16$ & $0.7 \pm--$ & $1.27 \pm 0.11$ & $1.34 \pm 0.25$ & $<0.001$ \\
\hline$\overline{\text { FS (\%) (2) }}$ & $28.8 \pm 4.4$ & $25.1 \pm 3.7$ & $25.0 \pm--$ & $32.0 \pm 3.7$ & $29.1 \pm 4.8$ & $<0.001$ \\
\hline$\overline{\mathbf{E F}(\%)(2)}$ & $55.1 \pm 7.2$ & $48.3 \pm 7.2$ & $45.0 \pm--$ & $59.4 \pm 5.3$ & $54.9 \pm 7.9$ & $<0.001$ \\
\hline$\overline{\mathbf{L A D}(\mathrm{mm})(2)}$ & $39.5 \pm 2.9$ & $42.2 \pm 2.2$ & $44.0 \pm--$ & $37.9 \pm 2.8$ & $39.6 \pm 3.2$ & $<0.001$ \\
\hline$\overline{\text { E/A ratio (2) }}$ & $0.99 \pm 0.9$ & $1.34 \pm 1.1$ & $3.2 \pm--$ & $0.89 \pm 0.4$ & $1.09 \pm 0.87$ & $<0.05$ \\
\hline Dec.time(msec)(2) & $182 \pm 51$ & $186 \pm 66$ & $110 \pm--$ & $193 \pm 46$ & $187 \pm 53$ & NS \\
\hline$\overline{\text { IVRT(msec) (2) }}$ & $108 \pm 26.2$ & $90 \pm 18.5$ & $\overline{70 \pm--}$ & $104 \pm 11.2$ & $100 \pm 19.6$ & $<0.05$ \\
\hline
\end{tabular}

$\mathrm{X} \pm$ SD: mean and standard deviation, WMSI: wall motion score index , FS:fractional shortening , EF: left ventricular ejection fraction, LAD: left atrial dimension, Dec. time: deceleration time, IVRT: isovolumic relaxation time.

By using the Paired Samples Statistics of echocardiographic data between hospitalization echo data* $1^{*}$ and after 6 months (follow up) echo data $* 2 *$ in group ( I,II and IV), table (5): as group III ( mortality group) were died before follow up echo done except one case.

Within group (I): The statistical analysis shows that there was significant difference between $(\mathrm{FS} 1 \& \mathrm{FS} 2)(\mathrm{P}$ value $=0.028)$ and $(\mathrm{LAD} 1 \&$ LAD2) $(\mathrm{P}$ value $=0.007)$ as FS decreased and LAD increased.

There was no significant difference in WMSI, EF , E/A ratio, Deceleration time and IVRT. (P value $>0.05$ ).

Within group (II): The statistical analysis shows that there was significant difference between (WMSI1\& WMSI2) ( $\mathrm{P}$ value $=0.002)$, $(\mathrm{FS} 1 \& \mathrm{FS} 2)(\mathrm{P}$ value $=0.002)$ and $(\mathrm{EF} 1 \& \mathrm{EF} 2)(\mathrm{P}$ value $=0.003)$ and (IVRT1\&IVRT2) (P value $=0.29)$ as FS and EF decreased and WMSI and LAD increased .

There was highly significant difference between $(\mathrm{LAD} 1 \& \mathrm{LAD} 2)(\mathrm{P}$ value $=0.000)$ as $\mathrm{LAD}$ increased.

There was no significant difference in E/A ratio and deceleration time ( $\mathrm{P}$ value $>0.05)$.
Within group (IV): The statistical analysis shows that there was significant difference between (EF1\&EF2) (P value=0.017) and $(\mathrm{LAD} 1 \& \mathrm{LAD} 2)(\mathrm{P}$ value $=0.004)$ as $\mathrm{EF}$ decreased and LAD increased .

There was no significant difference in WMSI, FS, E/A ratio, Deceleration time and IVRT. (P value $>0.05$ ). 
Table (5): Showed Paired Samples Statistics of echocardiographic data between hospitalization echo data*1* and after 6 months (follow up) echo data $* 2 *$ in group ( I,II and IV)

\begin{tabular}{|c|c|c|c|c|}
\hline Group & & & Paired $t$ & $\overline{\mathbf{P}}$ \\
\hline \multirow{14}{*}{ I } & \multirow{2}{*}{ Pair 1} & wmsi1 & .467 & .649 \\
\hline & & wmsi2 & & \\
\hline & \multirow{2}{*}{ Pair 2} & Fs1 & 2.472 & .028 \\
\hline & & Fs2 & & \\
\hline & \multirow{2}{*}{ Pair 3} & EF1 & 1.856 & .086 \\
\hline & & EF2 & & \\
\hline & \multirow{2}{*}{ Pair 4} & LAD1 & $-3.211-$ & .007 \\
\hline & & LAD2 & & \\
\hline & \multirow{2}{*}{ Pair 5} & EA1 & $-.067-$ & .947 \\
\hline & & $\overline{\text { EA2 }}$ & & \\
\hline & \multirow{2}{*}{ Pair 6} & Dect1 & $-.776-$ & .451 \\
\hline & & Dect2 & & \\
\hline & \multirow{2}{*}{ Pair 7} & IVRT1 & 1.124 & .281 \\
\hline & & IVRT2 & & \\
\hline \multirow{14}{*}{ II } & \multirow{2}{*}{ Pair 1} & wmsi1 & -3.832 & .002 \\
\hline & & wmsi2 & & \\
\hline & \multirow{2}{*}{ Pair 2} & Fs1 & 3.869 & .002 \\
\hline & & Fs2 & & \\
\hline & \multirow{2}{*}{ Pair 3} & EF1 & 3.629 & .003 \\
\hline & & EF2 & & \\
\hline & \multirow{2}{*}{ Pair 4} & LAD1 & $-4.516-$ & .000 \\
\hline & & LAD2 & & \\
\hline & \multirow{2}{*}{ Pair 5} & $\overline{\text { EA1 }}$ & $-1.626-$ & .126 \\
\hline & & EA2 & & \\
\hline & \multirow{2}{*}{ Pair 6} & Dect1 & .759 & .460 \\
\hline & & Dect2 & & \\
\hline & \multirow{2}{*}{ Pair 7} & IVRT1 & 2.431 & .029 \\
\hline & & IVRT2 & & \\
\hline \multirow{14}{*}{ IV } & \multirow{2}{*}{ Pair 1} & wmsi1 & 1.200 & .243 \\
\hline & & wmsi2 & & \\
\hline & \multirow{2}{*}{ Pair 2} & Fs1 & 1.773 & .090 \\
\hline & & Fs2 & & \\
\hline & \multirow{2}{*}{ Pair 3} & EF1 & 2.574 & .017 \\
\hline & & EF2 & & \\
\hline & \multirow{2}{*}{ Pair 4} & LAD1 & $-3.196-$ & .004 \\
\hline & & LAD2 & & \\
\hline & \multirow{2}{*}{ Pair 5} & EA1 & $-.716-$ & .481 \\
\hline & & $\overline{\text { EA2 }}$ & & \\
\hline & \multirow{2}{*}{ Pair 6} & Dect1 & .055 & .956 \\
\hline & & Dect2 & & \\
\hline & \multirow{2}{*}{ Pair 7} & IVRT1 & 1.409 & .173 \\
\hline & & IVRT2 & & \\
\hline
\end{tabular}

For the patients who had done coronary angiography were assessed by Gensini score. Table (6): Comparative analysis of Gensini score between the 4 studied groups :

The statistical analysis shows significant difference between groups $(\mathrm{p}<0.05)$, which was greatest in group II followed by group III then IV and I. And by Pearson correlation score ,there was highly significant positive correlation between MMP3 and Gensini which was 0.678 ( $\mathrm{p}$ value $=0.000)$. 
Table (6): Comparative analysis of Gensini score between the 4 studied groups :

\begin{tabular}{ccccccccc}
\hline & Group & No & Mean & Std. Deviation & Minimum & Maximum & F & P \\
\hline \multirow{2}{*}{$\begin{array}{c}\text { Gensini } \\
\text { score }\end{array}$} & II & 15 & 13.0667 & 6.71317 & 4.00 & 24.00 & 4.479 & $<0.05$ \\
\cline { 2 - 8 } & III & 3 & 34.3333 & 22.35770 & 6.00 & 72.00 \\
\cline { 2 - 8 } & IV & 9 & 16.7778 & 12.36707 & 6.00 & 36.00 \\
\cline { 2 - 7 } & Total & 33 & 18.8182 & 14.12766 & 4.00 & 72.00 & \\
\hline
\end{tabular}

As regard Pearson correlation between MMP3 and the other laboratory parameters there was :table (7)

* Highly significant positive correlation with cholesterol $(\mathrm{P}=0.003)$.
* Significant positive correlation with triglyceride $(\mathrm{p}=0.015)$.

* Significant negative correlation with HDL $(\mathrm{P}=0.021)$.

* Insignificant correlation with CBK, CK-MB, TN, LDl, AST and glucose.

Table (7): Shows Pearson Correlation between MMP3 and other laboratory parameters in the study population.

\begin{tabular}{lcl}
\hline & $\mathrm{r}$ & $\mathrm{P}$ \\
\hline CBK & 0.133 & $0.206(\mathrm{NS})$ \\
\hline CKMP & -0.110 & $0.298(\mathrm{NS})$ \\
\hline Tn & 0.182 & $0.082(\mathrm{NS})$ \\
\hline Thol. & 0.304 & $0.003(\mathrm{HS})$ \\
\hline HDL & 0.253 & 0.015 (Sig.) \\
\hline LDL & -0.243 & $0.021($ Sig.) \\
\hline AST & 0.175 & $0.100(\mathrm{NS})$ \\
\hline Glucose & 0.004 & $0.973(\mathrm{NS})$ \\
\hline Chi: che & 0.159 & $0.130(\mathrm{NS})$ \\
\hline
\end{tabular}

Chol: cholesterol , TG: triglycrides , HDL: high density lipoprotein , LDL: low density lipoprotein, AST: Aspartate transaminase

As regard Pearson correlation between MMP3 and echocardiographic data during hospitalization in the study population there was: table (8)

*Significant positive correlation with WMSI 1 $(\mathrm{p}=0.024)$.
*Insignificant positive correlation with EF1, LAD1, Dec. time1, IVRT1.

*Insignificant negative correlation with FS1 and E/A ratio 1.

Table (8): shows Pearson Correlation between MMP3 and echocardiographic data during hospitalization in the study population

\begin{tabular}{lcl}
\hline & $\mathrm{r}$ & $\mathrm{P}$ \\
\hline WMSI & 0.235 & $0.24(\mathrm{Sig})$. \\
\hline FSI & -0.089 & $0.398(\mathrm{NS})$ \\
\hline EF1 & 0.025 & $0.813(\mathrm{NS})$ \\
\hline LAD1 & 0.149 & $0.159(\mathrm{NS})$ \\
\hline E/A ratio1 & -0.008 & $0.943(\mathrm{NS})$ \\
\hline Dec. time 1 & 0.123 & $0.241(\mathrm{NS})$ \\
\hline IVRT1 & -0.036 & $0.735(\mathrm{NS})$ \\
\hline
\end{tabular}

WMSI:wall motion score index , FS:fractional shortening, EF: left ventricular ejection fraction, LAD: left atrial dimension, Dec. time: deceleration time, IVRT: isovolumic relaxation time 
As regard Pearson Correlation between MMP3 and echocardiographic data after 6 months (follow up) in the study population there was: table (9)

* Highly significant positive correlation with LAD2 ( $\mathrm{p}=0.001)$.

*Significant negative correlation with FS2 $(\mathrm{p}=0.019)$.
*Insignificant positive correlation with WMSI2, E/A ratio 2, Dec. time2 .

*Insignificant negative correlation with EF2 and IVRT2.

We used the ROC curve (area under curve $=0.781$, $\mathrm{p}=0.000)$ to identify the cut off point for the optimum combination of sensitivity and specificity for MMP-3 in the prediction of complications (morbidity and mortality).

Table (9): $\quad$ shows Pearson Correlation between MMP3 and echocardiographic data after 6 months (follow up) in the study population.

\begin{tabular}{lcc}
\hline & $\mathrm{r}$ & $\mathrm{P}$ \\
\hline WMSI2 & 0.246 & $0.075(\mathrm{NS})$ \\
\hline FS2 & -0.321 & $0.019(\mathrm{Sig})$. \\
\hline EF2 & -0.246 & $0.75(\mathrm{NS})$ \\
\hline LAD2 & 0.443 & $0.001(\mathrm{HS})$ \\
\hline E/A ratio2 & 0.059 & $0.673(\mathrm{NS})$ \\
\hline Dec. time 2 & 0.019 & $0.893(\mathrm{NS})$ \\
\hline IVRT2 & -0.182 & $0.193(\mathrm{NS})$ \\
\hline
\end{tabular}

WMSI:wall motion score index , FS:fractional shortening, EF: left ventricular ejection fraction, LAD: left atrial dimension, Dec. time: deceleration time, IVRT: isovolumic relaxation time

The cut off value of MMP3 was (67.6 $\mathrm{ng} / \mathrm{ml}$ ) with positive predictive value of $58.3 \%$ negative predictive value of $79.5 \%$, sensitivity of
$75.7 \%$, specificity of $63.6 \%$ and accuracy of $68.4 \%$.table (10)

Table (10)

\begin{tabular}{cccccc}
\hline Cut off value & Sensitivity & Specificity & $\begin{array}{c}\text { +ve predictive } \\
\text { value }\end{array}$ & $\begin{array}{c}\text {-ve predictive } \\
\text { value }\end{array}$ & Accuracy \\
\hline 67.6 & $75.7 \%$ & $63.6 \%$ & $58.3 \%$ & $79.5 \%$ & 68.4 \\
\hline
\end{tabular}

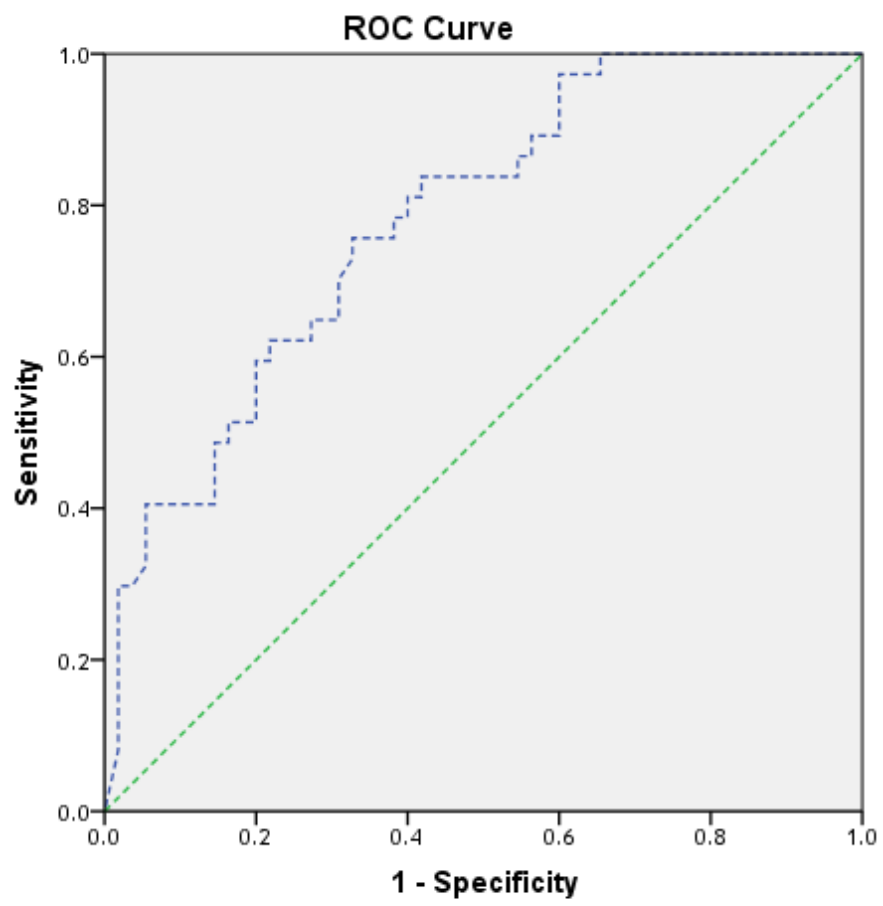

Diagonal segments are produced by ties. 


\section{DISCUSSION}

Following acute myocardial infarction (AMI) many cytokines and proteolytic enzymes are released. Among these, matrix metalloproteinases (MMPs) are important proteolytic enzymes that lead to degradation of the extracellular matrix and to change in cardiomyocytes in both infracted and noninfarcted myocardium. This process is known as cardiac remodelling. It has been demonstrated that more than one type of MMP is present in the circulation after cardiomyocye injury. A number of studies have demonstrated the correlation between these MMP levels and the severity of coronary lesion, the prognosis of left ventricular dimension and the survival rate following AMI ${ }^{(3)}$.

In this study, we examined the relationship of MMP-3 with LV remodelling and prognosis after AMI as assessed echocardiographically in addition to its relationship with the patients' age, sex, risk factors and laboratory findings.

We observed that there was significant difference in MMP3 level between groups $(\mathrm{p}<0.05)$, which was higher in morbidity and mortality groups more than the other groups this in agreement with Kelly et al. ${ }^{(7)}$.

We agree with Peter et al(13). who concluded that The apparent lack of correlation between MMP-3 and markers of the extent of myocardial necrosis, namely CK and Troponin I, may support the assertion that MMP-3 is an indicator of the remodelling which follows infarction, rather than simply a marker of the infarction itself As our study showed that there was insignificant correlation between MMP-3 and CBK, CKMB\&TN

Echocardiography: A two dimensional echocardiogram with Doppler analysis yields useful information about overall and regional left and right ventricular systolic and diastolic function, valvular integrity and function, pulmonary arterial systolic pressure, and the possible presence of pericardial effusion or ventricular septal rupture. The presence of moderate or severe right or left ventricular systolic or diastolic dysfunction significantly worsens prognosis and may lead the clinician to more aggressive diagnostic and therapeutic endeavors ${ }^{(\mathbf{8})}$.

We observed association between circulating MMP-3 and the degree of LV remodelling occurring after AMI, as assessed echocardiographically Adverse LV remodelling is one of the most prognostically significant consequences of AMI ${ }^{(11)}$. In this study we found that in the predischarge echo there was Significant positive correlation of MMP3 with WMSI 1 $(p=0.024)$, while negative correlations with FS and
IVRT \& in the follow up echo after 6 months there was negative correlation with FS2 $(\mathrm{p}=0.019)$, also with EF2 and IVRT2, while positive correlations with LAD2 ( $\mathrm{p}=0.001$ ), also with WMSI2, E/A ratio 2 and Dec. time 2. These findings were in agreement with the study findings of Kelly et al. ${ }^{(7)}$.

Our study showed that there was significant difference in Gensini score for coronary angiography between groups $(\mathrm{p}<0.05)$, which was higher in morbidity and mortality groups, and there was highly significant correlation between MMP3 and Gensini score, this in agreement with Samnegard et al. ${ }^{(12)}$ who proved that there was positive relationship between serum MMP-3 concentration and coronary artery disease severity.

Our study showed that there was increased risk of complications for patients in whom MMP-3 was above the cut off value which is in agreement with Kelly et al. ${ }^{(7)}$.

\section{ACKNOWLEDGEMENT}

We thank the patients for their kind participation in our study

\section{REFERENCES}

1. Boersma E, Pieper KS, Steyerberg EW, et al. predictors of outcome in patients with acute coronary syndromes without persistent STsegment elevation: results from an international trial of 9461 patients. Circulation 2000;101:255767.

2. Mori S, Gibson G, Mc Tiernan CF. Differential expression of MMPs and TIMPs in moderate and severe heart failure in transgenic model. J Card Fail 2006;12:314-25

3. Phatharajaree, W, Phrommintikul A, Chattipakorn N. Matrix metalloproteinases and myocardial infarction. Can J Cardial 2007;23(9):727-733.

4. Feigenbaum's. Echocardiography ... J Am Coll Cardiol 1999;33:512-521. Steinberg EH ... J Thorac Cardiovasc Surg 1999;118:542-546. Das MK, Pellikka PA, ...

5. Smart SC, Sawada S, Ryan T et al. Low-dose dobutamine echocardiography detects reversible dysfunction after thrombolytic therapy of acute myocardial infarction. Circulation 1993; 88:405415.

6. Taylor $\mathrm{CL}$ and Waggoner D. Iso-volumic Relaxation Time (IVRT) and diastolic dysfunction. J Am Soc Echocardiogr 1992;5:497-503.

7. Kelly D, Cockerill G, Ng LL, Thompson M. Circulating Stromelysin-1 (MMP-3): a novel predictor of LV dysfunction, remodelling and allcause mortality after acute myocardial infarction Eur J Heart Fail. Author manuscript; available in PMC 2009 February 3. Published in final edited form as: Eur J Heart Fail. 2008 February; 10(2): 133-139. Published online 2008 January 30. doi: 10.1016/j.ejheart.2007.12.009

8. Kober, L, Torp-Pederson, C, Pederson, OD, et al. On behalf of the TRACE Study Group. Importance of congestive heart failure and interaction of 
congestive heart failure and left ventricular systolic function on prognosis in patients with acute myocardial infarction. Am J Cardiol 1996; 78:1124.

9. Nijland, F, Kamp, O, Karreman, AJP, et al. Prognostic implications of restrictive left ventricular filling in myocardial infarction: Serial Doppler echocardiographic study. J Am Coll Cardiol 1997; 30:1618.

10. Daniel Chan and Leong L Ng. Biomarkers in acute myocardial infarction BMC Medicine 2010, 8: 34 doi:10.1186/1741-7015-8-34.
11. Pfeffer MA and Braunwald E. Ventricular remodeling after myocardial infarction. Experimental observations and clinical implications. Circulation 1990; 81:1161-1172.

12. Samnega A, Silveira A, Tornvall B et al. Lower serum concentration of matrix metalloproteinase-3 in the acute stage of myocardial infarction Journal of Internal Medicine 2006; 259: 530-536 doi:10.1111/j.1365-2796.2006.01632.x 